\title{
Arborescences
}

Revue d'études françaises

\section{La Seine du crime et les petits métiers parisiens}

\section{Nicolas Gauthier}

Numéro 8, décembre 2018

La Seine littéraire au XIX ${ }^{\mathrm{e}}$ siècle

URI : https://id.erudit.org/iderudit/1055882ar

DOI : https://doi.org/10.7202/1055882ar

Aller au sommaire du numéro

Éditeur(s)

Département d'études françaises, Université de Toronto

ISSN

1925-5357 (numérique)

Découvrir la revue

Citer cet article

Gauthier, N. (2018). La Seine du crime et les petits métiers parisiens.

Arborescences, (8), 33-45. https://doi.org/10.7202/1055882ar

\section{Résumé de l'article}

La Seine offre un défi particulier à l'auteur de mystères urbains dont le programme consiste à dévoiler les secrets de Paris : il paraît tout aussi incongru de faire l'économie de cet élément incontournable de la géographie parisienne que de présenter ce fleuve bien connu comme un mystère. Plusieurs de ces romans choisissent donc la seconde option, mais en proposant du fleuve une mise en scène complexe, organisée autour d'une population de personnages exerçant divers petits métiers présentés, eux, comme méconnus du lecteur. Y dominent les figures du chiffonnier et du pirate, livrant du monde fluvial un portrait d'abord socioéconomique. À travers l'analyse des modalités de cette mise en scène, cette étude vise à montrer comment les mystères urbains situent, dans la grande ville, l'espace que constitue la Seine. 


\section{SOMMAIRE}

1 Nicolas Gauthier, Université de Waterloo

Sébastien Roldan, chercheur indépendant

Introduction

6 Janine Gallant, Université de Moncton

La Seine de Mérimée et ses ressorts dramatiques

17 Silvia Baroni, Université de Bologne

Au seuil de l'enfer: la Seine dans La Comédie humaine

33 Nicolas Gauthier, Université de Waterloo

La Seine du crime et les petits métiers parisiens

46 Sébastien Roldan, chercheur indépendant

Où est la Seine dans les Tableaux parisiens de Baudelaire?

64 Valérie Narayana, Université Mount Allison

Géocritique du fleuve dans les mémoires de la Commune de Louise Michel

80 Peter Vantine, Saint Michael's College

Au bord de la Seine avec les Goncourt

102 Lola Kheyar Stibler, Université Sorbonne Nouvelle

«Hydrargyre» de Maurice de Fleury ou l'imagination de la matière 


\title{
La Seine du crime et les petits métiers parisiens
}

\author{
Nicolas Gauthier, Université de Waterloo
}

\section{Résumé}

La Seine offre un défi particulier à l'auteur de mystères urbains dont le programme consiste à dévoiler les secrets de Paris: il paraît tout aussi incongru de faire l'économie de cet élément incontournable de la géographie parisienne que de présenter ce fleuve bien connu comme un mystère. Plusieurs de ces romans choisissent donc la seconde option, mais en proposant du fleuve une mise en scène complexe, organisée autour d'une population de personnages exerçant divers petits métiers présentés, eux, comme méconnus du lecteur. Y dominent les figures du chiffonnier et du pirate, livrant du monde fluvial un portrait d'abord socioéconomique. À travers l'analyse des modalités de cette mise en scène, cette étude vise à montrer comment les mystères urbains situent, dans la grande ville, l'espace que constitue la Seine.

Frontière ne pouvant être traversée qu’à certains passages, dirigeant ou interrompant les déambulations, la Seine paraît inéluctable pour l'explorateur de Paris: qu'il soit touriste, flâneur ou détective, il s'y heurtera éventuellement. Il est de ce fait logique de la rencontrer dans les romans qui annoncent offrir un tableau de Paris. Il n'en reste pas moins que ce statut d'élément éminemment reconnaissable de la géographie parisienne peut aussi créer certaines ambiguïtés, comme c'est le cas au sein des «mystères urbains". Rappelons que ces romans, dont la "matrice» est Les Mystères de Paris d'Eugène Sue (Kalifa 2005: 40) - publié dans le Journal des débats du 19 juin 1842 au 15 octobre 1843 -, ont pour ambition de rendre visibles et lisibles les secrets de la cité, de la faire (re) découvrir au lecteur en montrant ce qui est caché, méconnu, c'est-à-dire les «mystères» de la ville ${ }^{1}$. Dans cette perspective, la Seine constitue un écueil pour les auteurs: elle semble inévitable mais faire un «mystère» de ce lieu bien connu des Parisiens ne va pas de soi. Néanmoins, plusieurs romanciers ont tout de même tenté de la représenter comme un espace crucial de leur Paris mystérieux, en posant que ce que voit l'observateur non initié n'est qu'une apparence trompeuse, et que la Seine n'est pas ce qu'elle paraît être.

C'est à l'étude des modalités de cette mise en fiction du fleuve qui traverse Paris que nous nous attacherons. Plus précisément, nous tenterons de mettre en lumière la façon dont différents mystères urbains résorbent la distinction entre la ville et le fleuve, le portrait de la première intégrant celui du second, en faisant un simple élément de la cité. Dans ce contexte, la Seine est analogue à la rue, autre composante incontournable de l'espace urbain: la singularité de l'une et de l'autre passe par leurs «habitants». En nous penchant d'abord sur ce que la Seine est et n'est pas dans ces romans, nous constaterons que l'enjeu crucial du portrait du fleuve est la représentation de ses habitants, organisée

1. On les compte par dizaines en France au XIX ${ }^{\mathrm{e}}$ siècle; les titres créent un véritable réseau puisque les auteurs ne modifient généralement que très partiellement celui de Sue: Les vrais mystères de Paris (Eugène-François Vidocq, 1844), Les Mystères du vieux Paris (Pierre Zaccone, 1854), Les Mystères du nouveau Paris (Fortuné du Boisgobey, 1876), Les nouveaux mystères de Paris (Aurélien Scholl, 1866). Dans plusieurs cas, le substantif "mystères" est remplacé - Les Mohicans de Paris (Alexandre Dumas, 1854-1859), Les Mendiants de Paris (Clémence Robert, 1848) -, mais le propos reste inscrit dans la lignée de celui de Sue, alors que dans d'autres cas, une translation géographique sert de point de départ: Les Mystères de Londres (Paul Féval, 1843-1844), Les Mystères de Marseille (Émile Zola, 1867). 
autour de deux figures: le chiffonnier et le pirate. Celles-ci font apparaitre des dynamiques essentielles de la mise en fiction de la Seine dans les mystères urbains, tout particulièrement celle consistant à afficher d'emblée une altérité que le récit transforme progressivement en une similarité. Au fil de ces analyses, nous observerons que le portrait de la Seine est inspiré non par des élans lyriques mais par des considérations prosaïques destinées malgré tout à participer à l'édification d'un mythe de Paris.

\section{Facettes de la Seine}

Parmi les nombreux mystères urbains ayant un cadre parisien au XIx ${ }^{e}$ siècle, plusieurs présentent différents passages conférant un rôle significatif à la Seine ${ }^{2}$. Nous concentrerons notre attention sur trois romans éloquents à cet égard: Les Mystères de Paris de Sue, Les vrais mystères de Paris, œuvre qu'EugèneFrançois Vidocq a publiée en sept volumes chez l'éditeur Cadot en 1844, et Les Mystères du nouveau Paris, que Fortuné du Boisgobey a fait paraitre chez l'éditeur Dentu en 1876. Dans Les Mystères de Paris, une intrigue associée à «l'île des Ravageurs", où vivent des criminels tirant leur subsistance de la Seine, s'étire sur sept chapitres non consécutifs, réunit plusieurs des personnages principaux (comme Fleur-de-Marie) et offre le cadre d'actions cruciales. Dans Les vrais mystères de Paris et Les Mystères du nouveau Paris, le lecteur rencontre plusieurs passages courts et dispersés qui présentent des péripéties liées à la Seine (utiliser le fleuve pour se déplacer secrètement ou échapper à des poursuivants, pour assassiner quelqu'un ou se débarrasser d'un cadavre, pour attenter à sa propre vie).

Ceci posé, il convient de parler d'abord de ce que la Seine n'est pas dans ces romans. Ceux-ci n'annoncent pas les élans poétiques que le fleuve suscitera plus avant dans le siècle. Lorsque Victor Hugo en 1862 décrit le suicide de Javert dans Les Misérables, il en offre le portrait suivant: «Le grossissement du fleuve plutôt dessiné qu'aperçu, le tragique chuchotement du flot, l'énormité lugubre des arches du pont, la chute imaginaire dans ce vide sombre, toute cette ombre était pleine d'horreur» (Hugo 1998: 1774). Le passage dont est tiré cet extrait multiplie les adjectifs et les métaphores et illustre comment la Seine peut faire vibrer la fibre poétique des écrivains après $1850^{3}$. Cependant, les mystères urbains, de la première ou de la seconde moitié du siècle, n'optent pas pour de longues descriptions de la Seine et sont avares de précisions quant à son aspect. À l'aune de ce passage tiré des Misérables, leurs descriptions paraissent minimalistes. Prenons un exemple tiré des Mystères du nouveau Paris: «La Seine, refoulée par la bourrasque et fouettée par l'averse, a des vagues courtes» (t. III, 122). Une telle retenue peut étonner, car les auteurs de mystères urbains ne craignent ni les descriptions grandiloquentes ni les envolées poétiques, tout particulièrement à propos des sites que visitent les personnages. Bien souvent, ces lieux sont présentés avec force détails, conformément au projet de dévoilement des mystères de la ville par le biais de l'exposition d'endroits révélateurs. Seulement, ces envolées ne concernent pas la Seine ${ }^{4}$.

2. Par exemple Les Mendiants de Paris (1848) de Clémence Robert (1797-1872), Les nouveaux mystères de Paris d'Aurélien Scholl (1833-1902) - publié dans Le Petit Journal du 2 octobre 1866 au 16 janvier 1867 - ou encore L'Héritage mystérieux, premier tome des Drames de Paris de Ponson du Terrail (1829-1871), publié dans La Patrie du 21 janvier au 4 octobre 1857.

3. Voir par exemple l'article de Peter Vantine dans le présent numéro à propos de la Seine chez les Goncourt.

4. Sans multiplier les exemples, mentionnons que, dans Les Mystères de Paris, la présentation de la famille de Ravageurs et de «leur» île (702-726) n'offre aucune description de la Seine, pas même lorsqu'un personnage se jette dans le fleuve pour nager jusqu'à cette île (850-859). Dans ce dernier cas, on ne trouve que des passages comme ceux-ci: «la Louve, éperdue de frayeur, transportée de rage, n'écoutant pas davantage le pêcheur, s'était encourue le long de la Seine» (854), "arracha son bonnet, laissa glisser sa robe jusqu’à ses pieds, ne garda que son jupon, se jeta 
Les romanciers récusent ou détournent d'autres associations qui pourraient aujourd'hui sembler aller de soi. Par exemple, la Seine, espace dynamique et de circulation souvent rapide - voire miraculeuse - dans le roman-feuilleton, devient dans notre corpus un lieu d'enfermement (dans des bateaux, à l'intérieur de maisons donnant sur la rive, dans des égouts, en des tunnels inondés par le fleuve, etc.). De même, bien que la Seine puisse être associée à la campagne, à la nature ou à des activités comme le canotage (on pense à Guy de Maupassant), dans les romans qui nous occupent, elle est récupérée par la ville et rarement considérée comme un lieu de villégiature. Apparemment espace public ouvert, la Seine se révèle surtout être un espace opaque aux non-initiés. En d'autres mots, ces mystères urbains construisent un portrait de la Seine qui cadre difficilement avec plusieurs procédés attendus, qui ne fait pas de ces derniers des principes structurants.

Bien évidemment, la Seine de ces romans n'est pas construite qu'en négatif. Retenons certaines caractéristiques qui paraissent cruciales. Tout d'abord, le fleuve joue un rôle de marqueur géographique destiné à permettre aux personnages - et au lecteur - de se situer dans cette grande ville labyrinthique. Dans Les vrais mystères de Paris, Vidocq multiplie les références aux quais: un personnage flâne jusqu'au "quai de la Mégisserie» (t. IV, 46), un autre doit échapper à la police en choisissant «le quai de Gèvres [ou] celui de la Cité» (t. IV, 60), des prostituées arpentent le «quai aux fleurs» (t. III, 247), et ainsi de suite. Ce qui est plus intéressant, c'est que cette fonction de repérage spatial fonctionne aussi au niveau social. Ces mystères urbains n'optent pas pour des axiomes généraux à propos de la Seine, comme le fait parfois Balzac - on se rappelle, par exemple, cette formule du Père Goriot: "Qui n'a pas pratiqué la rive gauche de la Seine, entre la rue Saint-Jacques et la rue des Saints-Pères, ne connaît rien à la vie humaine» (Balzac 1971 : 140). Il n'en reste pas moins que la Seine sert bel et bien à organiser le portrait de la société, selon des principes inattendus.

Avant d'examiner cette facette de la Seine, une autre remarque s'impose toutefois. Bien qu'elle puisse être présentée comme un lieu paisible ${ }^{5}$, elle est surtout connotée comme un espace menaçant, où n'évoluent que des criminels, et où l'on disparaît. Cette tendance n'est pas propre aux mystères urbains, comme l'a déjà remarqué Pierre Citron, qui explique que, sauf exception, «la Seine, ses quais, ses ponts [agissant comme] images par excellence de calme et de paix [...] ne se manifest[ent] guère lors des débuts du romantisme: la Seine est alors soit grandiose, soit boueuse, gémissante et truffée de cadavres» (1961: t. II, 175). Ajoutons que, à la lumière des analyses de Citron, le fleuve des mystères urbains semble évoquer tout particulièrement la Seine froide, sordide et lugubre souvent chantée par les poètes romantiques de la monarchie de Juillet (t. I, 423-426). Dans les mystères urbains, les malfaiteurs se servent du fleuve pour se dissimuler (en y plongeant), pour se faire oublier (en se cachant sur une île ou dans un lieu riverain peu fréquenté des autorités) ou pour faire disparaître une victime encombrante. Plusieurs techniques sont employées, par exemple dissimuler un cadavre mutilé dans un tonneau (Vidocq, Les vrais mystères de Paris: t. IV, 16-18) ou le "charriage à la mécanique", une manœuvre pour voler, tuer et faire disparaître la preuve en quelques secondes, généralement en la précipitant dans la Seine (Boisgobey, Les Mystères du nouveau Paris: t. I, 168-170 et 257). La diversité et l'efficacité de ces procédés, décrits à plusieurs reprises, assimilent les rives du fleuve à des coupe-gorge. Le phénomène est si répandu qu'il devient une des caractéristiques que les parodies de mystères urbains

intrépidement dans la rivière, y marcha tant qu'elle eut pied, puis, le perdant, elle se mit à nager vigoureusement vers l'île» (855).

5. Par exemple, dans Les Mystères de Paris, un personnage s'installe dans une petite maison tranquille retirée au bord de l'eau près d'Asnières (792). 
se plaisent à reprendre. Ainsi du récit «Voyage de M. Fitz-Gerald. À la recherche des mystères » de Léon Gozlan, paru dans la Revue de Paris en mai 1844, qui offre le portrait suivant du fleuve:

Les brouillards qui s'élèvent constamment de ce plateau sablonneux et de la Seine lui donnent la forme d'un chaos traversé par un pont; c'est celui d'Iéna. La vapeur en brise les arches, sous lesquelles il ne passe qu'un murmure triste et des bouffées grises. Au-delà, rien; à gauche, rien. Des soupçons de lumière, des apparences de bruit, des simulacres de choses, dansent et courent sous vos yeux comme les djins; horribles djins d'Occident, pâles, frileux, et qui vous dirigent vers un fossé où un assassin vous tue ignominieusement pour vingt sous avec un poignard rouillé et vous précipite ensuite dans la Seine afin de laver, non pas son crime, mais ses mains (Gozlan, «Voyage de M. Fitz-Gerald»: 14).

Ce passage synthétise d'une certaine façon le portrait de la Seine proposé dans les mystères urbains, en faisant toutefois intervenir un élan poétique qui fait défaut à la majorité d'entre eux, nous l'avons dit. Une telle mise en fiction de la Seine provient aussi de ce que, dans ces romans, un nombre impressionnant d'individus s'y enfoncent: les honnêtes personnages secondaires y sont précipités, les criminels et les héros y plongent pour fuir un agresseur ou pour sauver une victime. Si les premiers s'y noient, les seconds survivent, même lorsqu'ils se retrouvent en chemise dans la Seine presque gelée, parsemée de blocs de glace, durant de longues minutes, la nuit, au mois de décembre (Boisgobey, Les Mystères du nouveau Paris: t. I, 365-366). Tomber ou plonger dans la Seine devient ainsi une épreuve qui établit l'importance et le statut du personnage.

C'est aussi dire que le fleuve menaçant n'engloutit pas tout ce qu'on lui jette en pitance. Non seulement plusieurs personnages en ressortent vivants mais les corps qui y sont abandonnés ont aussi la fâcheuse habitude de réapparaître, malgré les précautions. De même, si le suicide par noyade dans la Seine est fréquemment évoqué, il est rarement mis en pratique: les désespérés optent pour d'autres méthodes. Javert s'enfonçant à jamais dans l'eau sombre pour disparaître du roman constituerait ici un contre-exemple ${ }^{6}$. Ceux qui tombent dans la Seine en ressortent, qu'ils soient bien vivants ou décédés et transportés pour être exhibés à la morgue, dont la visite devient un divertissement populaire pour les Parisiens. Une parenthèse s'impose ici. Vanessa R. Schwartz a étudié en détail ce phénomène (1999: 45-88), notant que, particulièrement après 1864, "les commentateurs ont décrit cette institution comme un site de plaisir et de divertissement - un "spectacle" " (59; nous traduisons). Elle suggère que les autorités ont travaillé de façon à "transformer la tâche sérieuse d'identification des corps en un théâtre gratuit pour les masses" (202; nous traduisons), inscrivant le tout dans un processus constituant "un nouveau type de foule» (202; nous traduisons). Il ne faut donc pas se surprendre du fait que la visite à la morgue devient également un motif littéraire abondamment employé (Boisgobey, Les Mystères du nouveau Paris: t. III, 117-118), associé dans l'imaginaire de l'époque à la Seine. Ajoutons enfin que, inapte à servir de tombeau pour les corps, le fleuve qui traverse Paris ne conserve pas davantage les objets qui lui sont confiés; ceux-ci réapparaissent, notamment grâce au labeur de multiples travailleurs dont le métier consiste à (re)pêcher ce qui est tombé dans le fleuve, sur ses rives ou dans les égouts. Cette deuxième vie des objets - et parfois des individus - est au cœur du monde bien organisé qui gravite autour de la Seine.

6. Cette situation fort rare n'est toutefois pas inexistante; c'est le cas, dans Les Mystères de Paris, de Mme Séraphin, servante du notaire Ferrand et complice de plusieurs de ses crimes, qui meurt noyée dans la Seine pour ne plus réapparaître (856). 


\section{Le(s) chiffonnier(s) de la Seine}

Car, en plus des facettes mentionnées précédemment, les romanciers font jouer à la Seine le rôle de centre de gravité pour une série de personnages pittoresques dont l'activité de subsistance principale dépend du fleuve. Ceux-ci sont présentés selon un mode familier à maints lecteurs du XIX ${ }^{e}$ siècle puisque les mystères urbains les rattachent explicitement aux petits métiers qui se déploient dans les rues parisiennes. C'est dire que le portrait qu'offrent ces œuvres de ce qu'il convient d'appeler les petits métiers de la Seine suit les mêmes axes que celui qu'elles proposent de leurs confrères explorant les artères de la ville. Ce faisant, elles transforment la Seine en rue parisienne.

Illustrant "un fragile équilibr[e] entre activité de survie et mendicité plus ou moins avouée» (Gourden 1992; cité dans Delattre 2003: 30977), les petits métiers sont très présents au XIX ${ }^{\mathrm{e}}$ siècle, dans la littérature et dans la sphère discursive en général. Simone Delattre, qui a abordé ce phénomène dans son ouvrage Les douze heures noires. La nuit à Paris au XIX siècle, offre un portrait détaillé de la situation des petits métiers, où on peut repérer trois axes qui structurent leur représentation. Il y a d'abord une dimension pittoresque, qu'exploitent souvent les romanciers en soulignant le caractère surprenant des tâches accomplies : aux cas bien connus des «chiffonniers, des hommes de la halle, des balayeurs ou des vidangeurs» (Delattre 2003: 309) s'ajoutent

le ramasseur de crottes de chiens, le ramasseur de bouts de cigares, [le] chasseur de rats et [le] chasseur de chats - animaux destinés, paraît-il, à être vendus sous le nom de gibelotte de lapin dans les gargotes populaires[, les] «ravageurs» et autres «regratiers [sic] de ruisseau " [qui, munis] d'un croc en fer ou d'un bâton, [f]ouillent les interstices qui séparent les pavés, dans l'espoir d'y découvrir des pièces ou des bijoux égarés, ou plus simplement des parcelles de métal propres à la revente [...] (Delattre 2003: 328-329).

Une telle nomenclature fait bien ressortir le large éventail de ces professions ignorées du grand public, ou présentées comme telles.

Cette méconnaissance provient également de ce que ces "professions» explorant la rue parisienne forment un "univers à part [...] déjà clos sur lui-même». Il s'agit du deuxième axe. Les membres de cet "univers à part» "fréquentent des bals et des débits de boissons qui leur sont réservés, pratiquent l'endogamie, et transmettent souvent leur métier à leurs enfants déguenillés» (Delattre 2003: 348), d'autant que leur "profession dégradante leur interdit toute intégration sociale, et les ravale au rang de parias» (348). Cette marginalisation se manifeste également dans une "propension au nomadisme urbain» (310), trait crucial faisant de ces individus une population difficile à circonscrire et, par le fait même, quelque peu inquiétante. Dominique Kalifa (2013: 52) et Simone Delattre (2003: 310), notamment, ont souligné le fait que l'imaginaire social rapproche petits métiers et criminalité, rapprochement explicite chez différents écrivains et commentateurs du $\mathrm{XIX}^{\mathrm{e}}$ siècle $^{8}$. Le phénomène s’explique

7. Il n'est pas inutile de rappeler également que, malgré leur marginalisation dans la seconde moitié du XIX ${ }^{\mathrm{e}}$ siècle (Delattre 2003: 347), les " "petits métiers" de la nuit ou de l'aube, indépendamment de leur représentation pittoresque, confèrent de la souplesse à l'organisation urbaine» (309). Ils fournissent également des formes de travail à différents membres de la famille: dans les milieux défavorisés, les "enfants apportaient leur contribution grâce aux petits métiers traditionnels, saute-ruisseaux, vendeurs de gâteaux, décrotteurs, ramoneurs, mendiants, puis gagnent à leur tour la fabrique» (Bernard 2007: 143-144).

8. En témoignent des écrits d'Eugène-François Vidocq (Quelques mots sur une question à l'ordre du jour, réflexions sur les moyens propres à diminuer les crimes et les récidives, Paris, Chez l'auteur, 1844), d'Émile de Labédollière (Les 
aisément par les traits qu'on retient des individus exerçant ces professions: nomadisme, marginalité, activités nocturnes échappant facilement à la surveillance ${ }^{9}$. Cette proximité avec l'univers criminel est le troisième axe du portrait des petits métiers.

Dans les mystères urbains qui nous occupent, le lecteur retrouve également cette structuration autour de ces trois axes lorsque sont abordés, parfois indirectement, les petits métiers. Le caractère pittoresque est mis de l'avant quand le narrateur des Mystères de Paris conduit son lecteur chez un receleur (751-756) ou au Temple, gigantesque bazar où l'on trouve de tout, sauf des articles neufs (477-479): dans les deux cas, il souligne que les marchandises proviennent soit d'individus endettés qui doivent vendre leurs biens, soit du travail et des trouvailles d'autres individus exerçant des petits métiers. On trouve une visite similaire dans Les Mystères du nouveau Paris (t. 3, 1-26), tandis que, dans le quatrième chapitre des Mohicans de Paris d'Alexandre Dumas, le narrateur aborde de front les petits métiers et introduit un groupe formé par un ouvrier, un ravageur des rues, un maçon, un charbonnier et un chasseur de chats (32-38). Dans le passage en question, la présentation des personnages donne à voir les trois axes (pittoresque, marginalité, criminalité) en quelques pages. Ainsi, les mystères urbains ne dérogent pas à une tendance répandue à l'époque. Cependant, il ne convient pas de nous arrêter trop longuement sur ces exemples, qui nous éloignent du fleuve.

Constatons plutôt que les mystères urbains qui donnent à voir des petits métiers associés à la Seine insistent également sur leur caractère pittoresque, leur marginalité et leur proximité avec l'univers criminel, comme nous allons l'observer. Lorsqu'il "présente» la Seine au lecteur, le narrateur des Mystères de Paris n'offre qu'une esquisse de paysage, mais il entreprend de formuler une nomenclature pittoresque de divers métiers, tels les «débardeurs» - «qui débarquent le bois flotté»-, les «déchireurs » - qui «démolissent les trains qui ont amené le bois» -, les «ravageurs» - qui "puis[ent] [...] le sable de rivière [pour en retirer] des fragments de bijoux d'or ou d'argent» (Les Mystères de Paris: $707^{10}$ ). Le narrateur des Mystères de Paris insiste sur ces derniers, présentés comme "plus criminels»; il attribue cette profession aux bandits appelés à participer activement aux péripéties. Fortuné du Boisgobey opte pour la même stratégie dans Les Mystères du nouveau Paris: son portrait de la Seine reste en premier lieu un tableau des professions y étant associées, notamment les "tafouilleux» - "chiffonniers de la Seine, [qui] écum[e]nt ses bords, ramass[e]nt les épaves et vol[e]nt au besoin» (t. I, 71) - et les "carapatas», "haleurs et déchireurs de bateaux» (t. I, 70). Survient aussi, dans plusieurs romans du genre, une autre profession: celle de "sauveteurs", payés par l'administration pour récupérer, morts ou vifs, les individus tombés dans la Seine (la récompense est nettement plus élevée lorsque la victime est sauvée, afin d'encourager les professionnels à se presser, leur célérité laissant parfois à désirer ${ }^{11}$ ). Le caractère pittoresque de ces professions est donc bien mis de l'avant.

Industriels, métiers et professions en France, Paris, Veuve L. Janet, 1842) et diverses ordonnances gouvernementales (Delattre 2003: 338-343).

9. Rappelons que «le métier du chiffonnier est de trouver, mais il ne lui est pas permis de tout s'approprier. En théorie, la loi [l'] oblige [...] à rapporter au commissariat de police le plus proche les objets de valeurs (billets, bijoux, couverts en argent jetés par erreur...) qu'il pourrait découvrir dans les rues» (Delattre 2003: 337). Cependant, la nuit, cette obligation est perçue comme moins contraignante... (Delattre 2003: 337).

10. Le terme est aussi utilisé pour désigner des individus qui, au moyen d'outils, fouillent les interstices entre les pavés (voir Delattre 2003: 328-329, déjà cité).

11. Par souci de clarté, citons Les Nuits du Palais-Royal (initialement intitulé Les Mystères du Palais-Royal), roman tout à fait comparable à ceux de notre corpus (mais où la Seine n'apparaît que plus rarement): «À Paris, l'autorité donne vingt-cinq francs de prime à quiconque sauve une personne en danger de se noyer; la prime n' est que de quinze francs si le sauveteur ne retire de l'eau qu'un cadavre» (Alonnier, Décembre et Raban, Les Nuits du Palais-Royal: 256). On 
De plus, les romanciers structurent les portraits des «habitants» de la Seine autour de leurs métiers de façon à en faire un groupe organisé quoique marginal par rapport à la société de Paris. Les narrateurs insistent ainsi sur des hiérarchies internes. Par exemple, celui des Mystères du nouveau Paris souligne que les tafouilleux se situent plus bas que les carapatas dans l'échelle sociale riveraine. Les confondre est un «faux pas» grave, un carapatas étant fort insulté qu’on le prenne pour un tafouilleux (t. I, 71). Le narrateur s'en moque, remarquant: «Où l'aristocratie va-t-elle se nicher!» Cette distinction n'empêche pas que les petits métiers de la Seine fassent l'objet du mépris du reste de la population dans ces romans (Sue, Les Mystères de Paris: 731-732; Boisgobey, Les Mystères du nouveau Paris: t. I, 54). Autrement dit, est mise en fiction ici une marginalisation conforme à celle que nous avons signalée plus haut.

Il apparaît également que ces professions ont maille à partir avec l'univers criminel. En fait, les individus honnêtes exerçant des petits métiers agissent presque systématiquement comme figurants, n'occupent pas de rôle significatif dans les péripéties. En contrepartie, ceux qui participent activement à l'intrigue sont des hors-la-loi. Après avoir décrit différentes professions associées à la Seine, c'est à raconter l'histoire de la famille de ravageurs criminels (voleurs et meurtriers) que le narrateur des Mystères de Paris se consacre, tandis que, dans Les Mystères du nouveau Paris, les riverains qui contribuent à l'intrigue sont tous des carapatas ou des tafouilleux, aussi voleurs les uns que les autres. Le narrateur des Vrais mystères de Paris, pour sa part, une fois évoquée l'existence de professions s'exerçant sur la Seine ou près d'elle, se tourne exclusivement vers ceux qui exploitent le fleuve pour leurs crimes.

La représentation des petits métiers de la Seine suit donc la même dynamique que celle des petits métiers pratiqués dans les rues de Paris; à cet égard, il n’y a pas de différence fondamentale entre l'espace fluvial et l'espace urbain. De la même façon que les autres lieux parisiens, la Seine est traitée dans ces romans comme un espace social, qui mérite qu'on en parle d'abord en raison de ses habitants.

Il n'en reste pas moins que plusieurs personnages exerçant des petits métiers font l'objet d'une valorisation. C'est le cas du chiffonnier, «figure de proue de tout un monde de petits ramasseurs qui font les délices de la description pittoresque» (Delattre 2003: $327^{12}$ ), en particulier parce que la

banalité extrême [de son] labeur nocturne [...] est rendue attractive par l'accumulation de détails individuels et d'anecdotes curieuses, puis érigée en spectacle presque épique, où ces travailleurs deviennent, collectivement, les éléments du cycle imperturbable par lequel, chaque nuit, Paris se nourrit et se nettoie (309).

en trouve une autre mention, moins explicite, dans Les Mystères du nouveau Paris (t. III, 117-118). On pense aussi au M. Dacheux de La Peau de chagrin. Raphaël de Valentin, au moment où il envisage de se suicider en se jetant dans la Seine, voit une «baraque surmontée d'un écriteau où ces paroles sont tracées en lettres hautes d'un pied: SECOURS AUX ASPHYXIÉS. M. Dacheux lui apparut armé de sa philanthropie, réveillant et faisant mouvoir ces vertueux avirons qui cassent la tête aux noyés, quand malheureusement ils remontent sur l'eau; il l'aperçut ameutant le curieux, quêtant un médecin, apprêtant des fumigations; il lut les doléances des journalistes écrites entre les joies d'un festin et le sourire d'une danseuse; il entendit sonner les écus comptés à des bateliers pour sa tête par le préfet de la Seine. Mort, il valait cinquante francs, mais vivant il n'était qu'un homme de talent sans protecteurs, sans amis, sans paillasse, sans tambour, un véritable zéro social. Inutile à l'État, qui n'en avait aucun souci " (Balzac, La Peau de chagrin: 33-34).

12. Déjà en 1831, Jules Janin l'indiquait sans ambages: "Dans les petits métiers, le chiffonnier est au moins le premier. Le chiffonnier est le plus grand des industriels en petit [...]»(1831: 341). Beaucoup plus près de nous, Antoine Compagnon ouvre ainsi un ouvrage récent intitulé Les Chiffonniers de Paris: «Le chiffonnier de Paris fut l'homme à tout faire, le maître Jacques du XIX ${ }^{\mathrm{e}}$ siècle, à la fois rôdeur inquiétant des faubourgs, agent essentiel des progrès de l'industrie, et figurant coloré des arts et des lettres» (2017: 9). 
Antoine Compagnon rappelle quant à lui que "le chiffonnier s'imposa bientôt comme une figure omniprésente de la vie littéraire, culturelle et artistique dès la Restauration et triompha sous la monarchie de Juillet» $(2017: 125)^{13}$. L'attrait des écrivains pour la profession s'explique aussi par le fait que «la collecte nocturne du chiffonnier peut revêtir quelque correspondance avec celle du littérateur: comme le poète, le chiffonnier explore la nuit parisienne en y espérant de l'inconnu» (330). Sous la plume de plusieurs auteurs, le chiffonnier serait en fait « détenteur d'une science toute particulière de la capitale, comme si la ville trahissait sa nature mauvaise dans ce qu'elle abandonne aux hasards des rues nocturnes» (Delattre 2003 : 335). Il devient ainsi «un symbole polyvalent, une métaphore participant au mythe de la ville» (Rietger 1988: 23), dont il paraît détenir la clé (Delattre 2003: 325). C'est dire que le chiffonnier s'impose au sein d'une catégorie de métiers qui constituent une occasion privilégiée d'organiser la description de la rue parisienne; il apparaît tout à fait riche pour l'écrivain prétendant dévoiler les secrets des artères de la cité et leur signification. Il en va de même à propos du fleuve.

Si les romans qui nous occupent ne proposent pas un type de chiffonnier qui serait pour la Seine l'équivalent de celui arpentant les rues parisiennes, tous les personnages associés à des petits métiers relevant du fleuve sont en fait un peu chiffonniers. La figure de celui qui (re)trouve des objets jetés ou perdus - ou qui les vole - et qui les revend ou en réutilise les composantes (tissu des vêtements, cuivre et autres métaux) est omniprésente; les métiers évoqués en sont des déclinaisons. Cette figure s'intègre ainsi à une visée déterminante des mystères urbains: dévoiler un circuit économique méconnu fondé sur une "deuxième vie" des objets. Comme les narrateurs s'arrêtent aux métiers qui sont à la limite de la légalité (ou situés de plain-pied dans l'illégalité), cette deuxième vie provient souvent de vols, ce qui situe également les transactions subséquentes et leurs profits dans un circuit économique distinct de l'économie officielle. Le portrait de la Seine s'inscrit de ce fait dans un plus large projet: présenter les ramifications d'une vaste économie parallèle, criminelle. Reprenons une observation de FrançoisEmmanuël Boucher à propos du roman balzacien et soulignons que ces romans déplacent «les discours économiques à l'extérieur des sphères qui leur seraient propres [jusqu’à] représenter le capitalisme industriel [ainsi que] la haute ou la basse spéculation d'une manière très différente de celle des économistes [...] contemporains" (Boucher 2013: 72). Les mystères urbains adoptent, pour traiter de la Seine, une perspective qui n'est ni poétique ni géographique mais qui est d'abord socioéconomique.

Le fleuve est mis en récit comme un endroit où on se débarrasse (infructueusement) d'objets (ou d'individus), qui nourrissent la population criminelle se chargeant de les récupérer. Dans ce contexte, où le portrait du cours d'eau passe par l'évocation de ces personnages, la figure du chiffonnier s'impose; mais parler de "chiffonniers sur l'eau» serait insuffisant. "[B] on sauvage que son caprice conduit à ne sortir que la nuit» (Delattre 2003: 325), le chiffonnier imaginaire est "partagé entre l'image du penseur débonnaire, presque dépourvu de besoins, et celle du nomade impossible à domestiquer" (Delattre 2003: 329). Enclins à miser sur la criminalité, les mystères urbains insistent sur la seconde image, celle du «nomade impossible à domestiquer». Pour les personnages associés à la Seine, une telle remarque appelle une autre figure connue, une figure dont l'apparition peut surprendre en contexte continental, celle du pirate.

13. À ce propos, on consultera également avec profit le numéro de la Revue d'Histoire littéraire de la France intitulé «Les chiffonniers littéraires» (2018). 


\section{De la piraterie au cœur de Paris}

Dans Les Mystères de Paris, comme dans Les Mystères du nouveau Paris, certains personnages sont assimilés explicitement à des pirates sévissant au cœur de la capitale française. Cela peut étonner en raison de l'exotisme traditionnellement attribué à la piraterie et du rapprochement que nous venons d'établir: si le chiffonnier est le plus souvent associé à la contemplation et à une certaine sérénité, le pirate "est la représentation nostalgique d'un fantasme de liberté et d'énergie» (Requemora-Gros 2014: 451). Néanmoins, Sue utilise l'expression "pirate d'eau douce»(Les Mystères de Paris: 713), tout comme Boisgobey, qui explique que les carapatas sont des "haleurs et déchireurs de bateaux, [de véritables] pirates d'eau douce» (Les Mystères du nouveau Paris: t. I, 70). Il ne faut pas y voir un calque de la formule facétieuse «marin d'eau douce». En l'occurrence, loin d'être inexpérimentés, ces pirates sont de véritables marins écumant la Seine, pillant les biens et enlevant d'innocentes victimes; les ravageurs de Sue coulent un petit bateau et les carapatas de Boisgobey font exploser une péniche. Bien que l'on ne parle pas ici de l'abordage d'un imposant navire par tout un équipage de pirates, ces épisodes prennent un aspect tragique et se soldent par des pertes de vies humaines. Sans écumer les vastes espaces de l'océan, ces personnages se livrent à une piraterie authentique.

Nous concentrer sur le fait que les romans présentent ces personnages comme des pirates est particulièrement fécond. En effet, cette perspective permet de mieux distinguer les ramifications économiques associées à la mise en récit de ces personnages et, partant, les enjeux au cœur de la représentation de la Seine. Commençons par rappeler que, durant la première moitié du Xix ${ }^{\mathrm{e}}$ siècle, le pirate possède une certaine actualité en ce qu'il est bien présent dans la sphère publique française. En raison de la "prise de conscience de la persistance de la piraterie", le gouvernement passa le 10 avril 1825 une loi qui «distingu[ait] les cas de piraterie commis lorsque la France était en état de paix ou en état de guerre», loi abrogée en 2007 seulement (Mathonnet 2014: 55). Sous la Restauration, «à mille lieues de l'image fantasmée et romantique de l'aventurier intrépide, anti-conformiste, du desperado ou autre révolté épris de liberté et chasseur de trésors perdus, l'image du pirate véhiculée par le droit est terrifiante»(Gariban 2008: $470^{14}$ ). Cependant, le XIX ${ }^{\mathrm{e}}$ siècle marque une mutation dans la figure du pirate; grâce aux progrès dans la navigation à vapeur, les choses changent: "Aussi longtemps que le quadrillage de l'océan Atlantique par les marines nationales demeurait incomplet, les pirates y proliférèrent. Dès le milieu du XIX ${ }^{\mathrm{e}}$ siècle, ce quadrillage est achevé, et c'en est dès lors fini de la piraterie transatlantique» (Keucheyan 2008: 467). En d'autres mots, il s'agit d'un premier basculement important. Il est vrai qu’à Paris, durant la première moitié du siècle, la piraterie maritime était une réalité connue de première main seulement des marins (de passage ou retraités) et des ouvriers et officiers de la marine fluviale (la Seine étant l'unique voie commerciale à communiquer avec l'océan). Cependant, cette piraterie maritime était tout de même associée à une certaine proximité; au milieu du XIX ${ }^{\mathrm{e}}$ siècle, il y a basculement, elle s'éloigne vers des mers et des océans plus exotiques, devenant une menace lointaine et peu courante.

La figure du pirate est également bien vivante dans la sphère littéraire, on le sait. Fort présente au XVIII ${ }^{\mathrm{e}}$ siècle - chez Defoe (pensons à son incontournable Histoire générale des plus fameux pirates,

14. Il n'est pas inutile de rappeler qu'un «véritable prolétariat maritime se forme dès la fin du XviI ${ }^{\mathrm{e}}$ siècle - peut-être le premier prolétariat au sens strict - qui est composé de paysans n'ayant pas trouvé à s'employer dans les grands centres urbains en voie de constitution" (Keucheyan 2008: 461-462). Ceci nourrit les rangs des pirates. Razmig Keucheyan appuie sa démonstration sur l'ouvrage Between the Devil and the Deep Blue Sea. Merchant, Seamen, Pirates, and the Anglo-American Maritime World, 1700-1750, de M. Rediker (2004). 
1724), Lesage, Voltaire et Marivaux (Requemora-Gros 2014: 448-449) -, elle devient encore plus visible dans la littérature romantique du premier XIX ${ }^{\mathrm{e}}$ siècle. On la retrouve entre autres sous la plume de Byron (Le Corsaire, 1814) et du jeune Balzac (Le Vicaire des Ardennes, 1822; Annette et le Criminel, 1824 - qui sera réimprimé en 1840 sous le titre Argow et le pirate). Mieux encore, le succès de James Fenimore Cooper (Le Corsaire rouge, 1828) inspira divers écrivains français, dont Édouard Corbière (Le Négrier, 1832) et Eugène Sue, qui fait paraitre pas moins de cinq ouvrages fortement influencés par le romantisme noir et dans lesquels la distribution fait la part belle aux pirates - notamment Kernok le pirate (1830) et Atar-Gull (1831 $\left.{ }^{15}\right)$. Dans ces œuvres, l'«image fantasmée et romantique de l'aventurier intrépide» évoquée par Gariban (470) est bien présente, mais elle n’a rien de statique, comme l'indique Sylvie Requemora-Gros:

C'est au milieu du XIX e siècle que le basculement romantique identifie le flibustier à l'imagerie de la bohème, avec des auteurs comme Gustave Aimard et Édouard Corbière, qui, cinquante ans avant Conrad, témoignent de l'héroïsation d'une figure de l'aventurier si décriée par Jules Verne et ses successeurs (2014: 450).

C'est ainsi que la figure du pirate est rapidement associée à la littérature médiatique et à la littérature pour la jeunesse (Harvey 2013: 149 et 159); «les pirates sont, tout au moins dans l'imaginaire collectif, des aventuriers. Ils incarnent le choix d'une vie audacieuse, de modes de subsistance novateurs, alternatifs, en rupture avec les normes sociales dominantes» (Keucheyan et Tessier 2008: 453). Après le passage d'une piraterie proche à une piraterie exotique et lointaine, ce second basculement dans la figure du pirate au $\mathrm{XIX}^{\mathrm{e}}$ siècle n'est toutefois pas encore complet dans les romans qui nous occupent. De fait, c'est un pirate qui «séduit, voire fascine» (Keucheyan et Tessier 2008: 453) qu’introduit Sue dans les mystères urbains, après avoir mis en scène cette figure à plusieurs reprises dans ses romans antérieurs.

Dans les mystères urbains qui forment notre corpus, le pirate d'eau douce évoluant sur la Seine crée une distance, inscrit un certain exotisme du fleuve. "[A]rchétype figurant la marginalité» (Harvey 2013: 158), le pirate rejoint le chiffonnier dans la revendication d'un statut extérieur à la société, ne s'y intégrant pas et n'en suivant pas les lois. Le narrateur des Mystères de Paris indique que les membres de la famille de ravageurs, dont le père a été exécuté et l'aîné envoyé au bagne, sont "en révolte ouverte[,] en guerre acharnée contre la société, et croient par de nouveaux crimes se venger de la juste punition qui a frappé eux ou les leurs» (737). Comme les carapatas, ils ne cherchent aucunement à s'intégrer à la société - ni à y vivre tranquillement des profits de leurs crimes -, mais l'attaquent par leurs méfaits afin de s'en dissocier.

C'est là, précisons-le, une revendication, non le reflet d'une autonomie réelle face à la société parisienne. Ces personnages sont en cela similaires au

pirate [des mers qui] ne peut donc exister sans système social à piller; il vit grâce à la société, tout en refusant d'en faire partie, d'être assujetti à ses lois. Il parasite le système, en s'enrichissant aux dépens d'autrui. Il n'est pas ermite: sa rupture avec la société n'est pas absolue mais circonstanciée. En d'autres termes, le pirate est un profiteur. Il profite d'abord des failles: celles du capitalisme, celles de la guerre (Keucheyan et Tessier 2008: 451-452).

15. Pour un portrait plus détaillé, nous renvoyons à l'ouvrage The Novel and the Sea de Margaret Cohen (2010), tout particulièrement au chapitre 4, intitulé "Sea Fiction in the Nineteenth Century: Patriots, Pirates and Supermen" (133-178). 
Le pirate comme "profiteur» : cette facette prédomine dans les mystères urbains analysés ici. Le narrateur des Mystères de Paris détaille comment l'un des ravageurs a volé des caisses sur un bateau et comment il partage ensuite le butin avec différents membres de sa famille (717-719). De façon similaire, les carapatas des Mystères du nouveau Paris enlèvent un homme, pillent ses possessions et cherchent ensuite à le rançonner (t. III, 110-114). Ces criminels, qui se déplacent sur l'eau et utilisent des bateaux, offrent aussi leurs services aux malfaiteurs ne possédant pas d'expertise fluviale, lorsque vient le temps d'assassiner sur la Seine un adversaire ou un témoin gênant (Sue, Les Mystères de Paris: 722-723). Ces crimes n'ont en soi rien d'inattendu mais laissent bien voir le caractère parasitique de ces personnages, de même que leur dépendance aux relais des réseaux économiques, légaux et illégaux, qu'utilisent les autres criminels représentés dans les mystères urbains. Rappelons que ceux-ci déploient beaucoup d'efforts pour mettre en scène cette circulation de biens et de fonds (Gauthier 2017a, 2017b). Pour s'enrichir du butin trouvé dans la caisse, les ravageurs doivent avoir recours à un receleur; pour rançonner leur victime, les carapatas doivent utiliser une banque et un receleur. Malgré la marginalité qu'ils revendiquent, ces pirates participent aux réseaux économiques criminels que mettent en relief les mystères urbains dans leur portrait de Paris.

En fait, perpétuellement en quête de butin, ces pirates d'eau douce, comme les pirates maritimes, représentent le "produi $[\mathrm{t}$ ] terrifian $[\mathrm{t}$ ] des États [qui sont] incapables d'assurer du travail aux plus démunis» (Harvey 2013: 153 ${ }^{16}$ ). Le caractère «terrifiant» de ces figures dérive aussi d'une ambiguïté touchant leur statut idéologique:

La définition même de la piraterie implique le brigandage et le vol de la propriété d'autrui. Or chez les pirates, les expropriations se font dans un but d'enrichissement personnel évident[,] sous-tendu par une valorisation de la richesse. Les attentats à la propriété commis par les pirates ne procèdent pas d'une quelconque critique de cette dernière, du type de celle à laquelle se livreront les anarchistes ultérieurement. Ils visent plus simplement, à la faire changer de main. Sur le plan idéologique, la piraterie a partie liée avec le libéralisme naissant (Keucheyan 2008: 468).

De fait, les auteurs de mystères urbains introduisent dans la Seine, au cœur de Paris ou dans ses environs, des figures connotant un certain exotisme et revendiquant une certaine marginalité, mais ils les intègrent parfaitement dans leur portrait critique de la quête incessante de profit qui anime toute la capitale. Il s'agit en fait d'un phénomène récurrent dans ces romans, que l'on peut tout autant observer à propos des cabarets mal famés (Gauthier 2017b). Les scènes présentant ces pirates donnent lieu à certaines remarques dénonçant les disparités sociales. Néanmoins, les auteurs ne formulent pas de critique portant sur les principes mêmes du capitalisme ou sur la notion de profit. Comme l'indique Keucheyan, l'enjeu concerne ici la distribution des richesses, et les pirates de la Seine ne cherchent pas à modifier le fonctionnement de la société; ils tentent plus simplement de s'enrichir en en contournant ou en en brisant les règles et les lois.

Comme le chiffonnier, les pirates de la Seine donnent à voir au lecteur une révolte violente mais en fin de compte vaine. La Seine devient, comme la rue parisienne, l'espace de personnages marginaux, parfois méprisables mais associés à une rébellion, thème cher au romantisme. Apportant une certaine poésie au portrait du fleuve, le chiffonnier et le pirate s'opposent explicitement à l'ouvrier

16. Razmig Keucheyan estime que les «pirates sont à rapprocher de ce que Éric Hobsbawm [dans Les Bandits] appelle les bandits sociaux [et qu'ils] émergent dans les phases de transition entre systèmes sociaux, notoirement au cours de la transition entre le féodalisme et le capitalisme, et lors des périodes de crise économique» (2008: 466). 
par la liberté dont ils semblent bénéficier, alors que celui-ci est confiné à un lieu de travail précis et à l'obéissance hiérarchique. Cependant, les apparences peuvent tromper. Le chiffonnier est "le maître dérisoire d'une nuit qu'en réalité, personne ne lui envie» (Delattre 2003: 326), tandis que le pirate est «en un sens [...] pleinement intégr[é] à la logique marchande» (Keucheyan 2008: 460). Keucheyan ajoute que, "bien que la piraterie contienne une charge anti-capitaliste indéniable, sa participation à la logique de l'accumulation est une possibilité qui s'est souvent actualisée au cours de l'histoire» (2008: 469). Revendiquant leur marginalité et se situant aux franges de la société, ces pirates d'eau douce obéissent aux mêmes logiques et présentent les mêmes travers que les criminels de ces romans associés au «centre» de la cité parisienne. Le fleuve est utilisé par les romanciers pour représenter une criminalité capitaliste fondée sur le "travail», une caractéristique qui se rapporte en fait à l'ensemble de la criminalité urbaine que proposent ces romans (Gauthier 2017a). À cet égard, à travers ces personnages - pirate comme chiffonnier -, la Seine constitue bien une sorte de prolongement des rues parisiennes, un espace fluvial mis en récit d'abord dans une perspective socioéconomique.

Loin d'être une occasion manquée, la mise en scène de la Seine dans les mystères urbains que nous avons examinés est riche et féconde. Les romanciers ont surmonté l'obstacle que constituait le caractère bien connu de la Seine pour une majorité de lecteurs en faisant de celle-ci non seulement un cadre et un repère spatial et social, mais aussi un lieu auquel est associée toute une panoplie de petits métiers. Ceux-ci servent à illustrer des relations complexes entre d'apparents marginaux, libres de toutes contraintes et résistant aux lois, et «la société" à laquelle ils prétendent s'opposer.

En basant leur portrait de la Seine sur les personnages que nous avons présentés, tout particulièrement le chiffonnier et le pirate, les romanciers font du fleuve un espace urbain analogue aux autres espaces parisiens qu'ils décrivent, comme si la ville absorbait le cours d'eau la traversant. Ici, le fleuve ne représente pas un lieu associé à la nature ou à une extériorité par rapport à la cité, bien au contraire. Et s'il y a poétisation de la Seine, celle-ci s'effectue en creux, par le biais de quelques remarques éparses mais surtout par la présentation de ses habitants pittoresques, de leurs destins tragiques et de leurs révoltes futiles. Plus que l'objet d'une contemplation, la Seine est un élément intégré dans le portrait d'une ville conçue comme un espace social organisé selon une perpétuelle et insatiable quête de profit. En d'autres mots, la Seine est d'abord et avant tout un argument dans une démonstration à laquelle les mystères urbains ramènent tout ce qu'ils mettent en scène.

\section{Références bibliographiques}

Alonnier, E., J. Décembre et L.-F. Raban. 1869. Les Nuits du Palais-Royal. Paris: Librairie Décembre-Alonnier. Balzac, H. 1971. Le Père Goriot. Paris: Gallimard. [1835].

Balzac, H. 1974. La Peau de chagrin, préface d'André Pieyre de Mandiargues. Paris: Gallimard. [1831].

Bernard, C. 2007. Penser la famille au dix-neuvième siècle, 1789-1870. Saint-Étienne: Presses universitaires de Saint-Étienne.

Boisgobey, F. 1876. Les Mystères du nouveau Paris. Paris: Dentu.

Boucher, F.-E. 2013. "Balzac, les espèces sociales et la représentation du pouvoir économique au XIXe siècle». Dans Fiction et économie. Représentations de l'économie dans la littérature et les arts du spectacle, XIX-XXI siècles, sous la direction de G. Sicotte, M. Poirson, S. Loncle, et C. Biet. Québec: Presses de l'Université Laval: 61-77. 
Citron, P. 1961. La Poésie de Paris dans la littérature française de Rousseau à Baudelaire. Paris: Minuit.

Cohen, M. 2010. The Novel and the Sea. Princeton: Princeton University Press.

Compagnon, A. 2017. Les Chiffonniers de Paris. Paris: Gallimard.

Compagnon, A. (dir). 2018. "Les chiffonniers littéraires", Revue d'Histoire littéraire de la France 3 (118 année).

Delattre, S. 2003. Les douze heures noires. La nuit à Paris au XIX siècle. Paris: Albin Michel. [2000].

Dumas, A. 1998. Les Mohicans de Paris. Paris: Gallimard. [1854-1859].

Gariban, S. 2008. «Hostes humani generis: les pirates vus par le droit». Critique 733-734: 470-479.

Gauthier, N. 2017a. Lire la ville, dire le crime. Limoges: Presses de l'Université de Limoges.

Gauthier, N. 2017b. «Le tapis-franc criminel et le salon respectable: mise en regard chronotopique dans les mystères urbains (1842-1859)». Nineteenth-Century French Studies, 46 (1-2): $42-57$.

Gourden, J.-M. 1992. Le Peuple des ateliers: les artisans du XIX siècle. Paris: Créaphis.

Gozlan, L. 1844. "Voyage de M. Fitz-Gerald. À la recherche des mystères». Revue de Paris 5: 5-28.

Harvey, S. 2013. "Commerce et circulation de marchandises et d'idées: la piraterie dans tous ses états». Dans Fiction et économie. Représentations de l'économie dans la littérature et les arts du spectacle, XIX' ${ }^{e}-X X I^{e}$ siècles, sous la direction de G. Sicotte, M. Poirson, S. Loncle, et C. Biet. Québec: Presses de l'Université Laval: 149-169.

Hobsbawm, É. 1999. Les Bandits. Paris: La Découverte.

Hugo, V. 1998. Les Misérables. Paris: Librairie générale française. [1862].

Janin, J. 1831. «Les petits métiers». Dans Paris, ou Le Livre des cent et un. Paris: C. Ladvocat: t. III, 317-342.

Kalifa, D. 2005. Crime et culture au XIX ${ }^{e}$ siècle. Paris: Perrin.

Kalifa, D. 2013. Les Bas-fonds. Histoire d'un imaginaire. Paris: Seuil.

Keucheyan, R. 2008. «Philosophie politique du pirate». Critique 733-734: 458-469.

Keucheyan, R. et L. Tessier. 2008 «Présentation. De la piraterie au piratage». Critique 733-734: 451-457.

Mathonnet, D. 2014. «L'évolution du droit de la piraterie en France de la période moderne à la loi du 20 décembre 2007 ». Dans La Piraterie au fil de l'histoire. Un défi pour l'État, sous la direction de M. Battesti. Paris: Presses de l'Université Paris-Sorbonne: 49-62.

Ponson du Terrail. A. 1857. L'Héritage mystérieux. Publié en feuilleton dans La Patrie (21 jan.-4 oct.).

Rediker, M. 2004. Between the Devil and the Deep Blue Sea. Merchant, Seamen, Pirates, and the Anglo-American Maritime World, 1700-1750. Cambridge: Cambridge University Press.

Requemora-Gros, S. 2014. "Généalogie de la figure littéraire du pirate du XVII ${ }^{\mathrm{e}}$ au XIx siècle». Dans La Piraterie au fil de l'histoire. Un défi pour l'État, sous la direction de M. Battesti. Paris: Presses de l'Université ParisSorbonne: 439-451.

Rietger, D. 1988. «"Ce qu’on voit dans les rues de Paris” : marginalités sociales et regards bourgeois». Romantisme 59: 19-29.

Robert, C. 1856. Les Mendiants de Paris. Paris: G. Roux.

Roche, D. 1981. Le Peuple de Paris. Essai sur la culture populaire au XVIII siècle. Paris: Aubier.

Scholl, A. 1867. Les nouveaux mystères de Paris. Paris: Librairie internationale.

Schwartz, V. R. 1999. Spectacular Realities. Early Mass Culture in Fin-de-Siècle Paris. Berkeley: University of California Press.

Sue, E. 1989. Les Mystères de Paris. Paris: Robert Laffont. [1842-1843].

Vidocq, E. 1844. Les vrais mystères de Paris. Paris: Cadot. 